1

\title{
Design of steel beams with discrete lateral restraints
}

\author{
Finian McCann ${ }^{a, *}$, Leroy Gardner ${ }^{a}$ and M. Ahmer Wadee ${ }^{a}$ \\ email: finian.mccann07@imperial.ac.uk; leroy.gardner@imperial.ac.uk; a.wadee@imperial.ac.uk \\ ${ }^{a}$ Department of Civil and Environmental Engineering, Imperial College London, London SW7 2AZ, \\ United Kingdom \\ *Corresponding author. Tel: +44 7517477765
}

\begin{abstract}
Discrete lateral restraints offer an effective means of stabilizing beams against lateraltorsional buckling. Design expressions for simply-supported beams braced regularly along their span with elastic restraints, based on analytically-derived formulae, are presented herein. These include the minimum restraint stiffness required to force the beam to buckle in between the restraint nodes and the forces induced in the restraints, along with a brief treatment of the critical moment of the beam. It is demonstrated that there is close agreement between the values obtained from the design formulae and their original analytical counterparts. These are also compared with the results from design formulae based on analogous column behaviour, an approach commonly used in design codes. It is found that the column rules used by design codes return values that, when compared with the results of the current analysis, are overly conservative for cases where the restraints are positioned at the compression flange of the beam but unsafe for restraints positioned at the shear centre.
\end{abstract}

Keywords: steel beams; discrete lateral restraints; beam stability; steel beam design 


\section{Manuscript \\ Click here to view linked References}

\section{Introduction}

Slender beams are susceptible to failure through lateral-torsional buckling, an instability phenomenon involving both lateral deflection and twist of the cross-section of the beam. The classical result for the critical lateral-torsional buckling moment of an unrestrained simply-supported beam under constant bending moment, as given by [1], is:

$$
M_{\mathrm{ob}}=\frac{\pi^{2} E I_{z}}{L^{2}} \sqrt{\frac{I_{w}}{I_{z}}+\frac{L^{2} G I_{t}}{\pi^{2} E I_{w}}} .
$$

where the material properties $E$ and $G$ are the Young's modulus and elastic shear modulus, respectively, of steel; the cross-sectional properties $I_{z}, I_{w}$ and $I_{t}$ are the minor-axis second moment of area, the warping stiffness and the St. Venant's torsional constant, respectively.

The stability of a beam can be enhanced through the provision of bracing members that restrain either one, or both, of these forms of displacement, thus increasing the overall load that the beam can safely support. Restraints can be continuous, like profiled metal sheeting, or discrete, like roof purlins. If they inhibit the amount of twist then they are described as torsional braces; if they inhibit lateral deflection, they are described as lateral braces. The current work focusses on beams with discrete lateral braces.

The British Standard BS 5950 [2] states that a cross-section can be assumed to be restrained laterally if the intermediate restraint at that section is sufficiently stiff to inhibit any lateral deflection of the compression flange relative to the supports. Moreover, BS 5950 states that, for one or two restraints, the members should be able to withstand a total force of $2.5 \%$ of the maximum compressive force in the beam, divided amongst the restraints in proportion to their spacing; for three or more restraints, each restraint should be able to withstand $1 \%$ of the maximum compressive force (or the $2.5 \%$ force distributed in proportion to the restraint spacing). Meeting these requirements allows the designer to divide the beam into segments with pin-joints assumed to exist at the bracing points. These segments are then checked individually to ensure stability throughout the beam.

The American Institute of Steel Construction Specifications for Structural Steel Buildings [3] primarily requires that lateral bracing members are to be attached at the compression flange of the beam (or to both flanges if the beam is in double curvature); the scope of the current work exteneurocodeds to braces located at an arbitrary height relative to the shear centre of the beam. The finite element analysis of [4] showed the negative effect of web distortion on the efficacy of lateral restraints, and so it is assumed in the current work that adequate web-stiffening is provided at bracing nodes. The cross-section of the beam at these points may hence be assumed to be rigid and that Vlasov conditions prevail. With regard to the strength of the braces, the AISC specifications require that they must be able to withstand an equivalent of $2 \%$ of the maximum compressive force in the beam for beams in single curvature (4\% for restraints beside an inflection point for beams in positive and negative curvature in the same span). The minimum stiffness requirement for nodal bracing, using the notation of the current work, is:

$$
K=\frac{1}{0.75} \frac{4 M}{L_{u} h_{s}}
$$

where $K$ is the restraint stiffness, $M$ is the maximum moment in the beam, $h_{s}$ is the distance between the centroids of the flanges and $L_{u}$ is the unbraced length of the beam. Interestingly, the formulation suggests that providing additional braces to a particular beam, which would reduce the unbraced length, leads to an increase in brace stiffness requirements.

The Eurocode EN 1993-1-1 [5] is less prescriptive than BS 5950 and the AISC specifications, merely stating that beams with sufficient restraint to the compression flange are not susceptible to lateral-torsional buckling and that the elastic critical moment should take into account (amongst other things) the influence of lateral restraints, thus leaving the designers to decide whether to divide the beam into laterally-unrestrained segments, or to use a method of determining the elastic critical moment of a laterally-restrained beam. It does not specify one particular restraint force 
ratio that the bracing members must be able to withstand, but instead, an equivalent stabilizing force based on the initial imperfection of the beam is determined, and the bracing system as a whole should be able to withstand this. For one member restrained, assuming the initial imperfection to be $L / 500$, this rule equates to a distributed restraint force ratio of at least $1.6 \%$, depending on the stiffness of the bracing system. The $1.6 \%$ value is a lower bound, since it is based upon the assumption that the restraining system does not deflect. Previous work presented in [6], [7] and [8] suggest that a figure closer to $1 \%$ is sufficient, and that members with this level of strength can be assumed also to possess a level of stiffness adequate to restrain the bracing nodes fully; this assumption, however, does not hold for all geometries and bracing layouts, particularly where bracing is provided closer to the shear centre of the beam.

Both the British and European design codes fail to provide a method of determining directly the stiffness required of the restraint to ensure that buckling occurs between the restraints, while the American specifications are formulated for restraint provided at the compression flange. Methods to determine the required stiffness for specific cases are detailed by [9], amongst others. It is therefore the aim of the current work to provide design formulae for beams with an arbitrary number of restraints spaced at regular intervals, positioned at an arbitrary height above the shear centre.

\section{Analytical method and results}

A linear Rayleigh-Ritz analysis is used to determine the critical moment and deflected shape of a simply-supported doubly-symmetric I-section beam of span $L$, with a number $n_{b}$ of discrete elastic lateral restraints, of stiffness $K$, positioned at regular intervals along the span of the beam, such that the restraint spacing $s=L /\left(n_{b}+1\right)$ (see Figure 1 ). The restraints are all positioned at a height $a$ above the shear centre of the cross-section; positive values of $a$ denote compression side bracing, while negative values denote tension side bracing. The beam is loaded with majoraxis end moments of magnitude $M$ acting in opposite senses. In keeping with classical analyses such as those of [10] and [1], the system is assumed to have two degrees-of-freedom: the lateral displacement of the shear centre, $u$ and the angle of twist of the cross-section, $\phi$; these are modelled as Fourier sine series, with the coefficients of cosine terms set equal to zero to satisfy the boundary conditions of zero deflection and zero twist at the supports:

$$
\begin{aligned}
& u=\sum_{n=1}^{\infty} u_{n} \sin \left(\frac{n \pi x}{L}\right), \\
& \phi=\sum_{n=1}^{\infty} \phi_{n} \sin \left(\frac{n \pi x}{L}\right),
\end{aligned}
$$

where $u_{n}$ and $\phi_{n}$ are the generalized coordinates of the system. The initial lateral imperfection of the beam is represented by a single half-sine wave of amplitude $e_{1}$, i.e. $e=e_{1} \sin (\pi x / L)$. Since the system is linearized by assuming small deflections and modelling the restraints as linear springs, the highest order of terms in $u_{n}$ and $\phi_{n}$ in the potential energy functional of the system, $V$, is two.

The deflected shape of the beam is determined by solving the simultaneous equilibrium equations $\partial V / \partial u_{n}=0$ and $\partial V / \partial \phi_{n}=0$ for $u_{n}$ and $\phi_{n}$. From these expressions it is possible to calculate the forces induced in the restraints.

The critical moment of the beam is determined by solving $\operatorname{det}(\mathbf{H})=0$ for $M$, where $\mathbf{H}$ is the matrix of the second derivatives of $V$ with respect to the generalized coordinates; these derivatives are equivalent to the coefficients of the second order terms of $V$ because of the linear nature of the system. Analysis of the system shows that the possible buckling modes separate into two classes: a finite number $n_{b}$ of node-displacing modes and an infinite number of modes where the beam buckles in between the restraints, which are related to harmonics that are integer multiples of $\left(n_{b}+1\right)$. A solution for the critical moment for such modes - termed internodal modes - is 
easily-obtainable; the most critical mode is that of a beam buckling in $n_{b}+1$ half-sine waves and so the associated critical moment, $M_{T}$, is equivalent to the critical moment of an unrestrained beam segment of span $s$ :

$$
M_{T}=\frac{\pi^{2} E I_{z}}{s^{2}} \sqrt{\frac{I_{w}}{I_{z}}+\frac{s^{2} G I_{t}}{\pi^{2} E I_{w}}} .
$$

This moment is termed the threshold moment. In the case of the node-displacing modes, however, it is not possible to find a closed-form solution for $M$, but instead an implicit relationship between $M$ and the coordinates can be determined [11].

An unrestrained beam, i.e. where $K=0$, buckles into a single half-sine wave. As $K$ is increased, the critical mode changes, as shown in Figure 2. If the restraints possess adequate stiffness, the beam buckles in between the restraints, i.e. the internodal buckling mode is critical and hence the critical moment equals $M_{T}$. This occurs if the stiffness $K$ exceeds the threshold stiffness $K_{T}$; this level of bracing is termed full bracing. If $K<K_{T}$, then one of the node-displacing modes is critical. The value of $K_{T}$ for the $m$ th node-displacing mode is determined by substituting $M_{T}$ into the implicit load-deflection relationship for that mode and determining the conditions necessary for an infinite deflection, a condition associated with critical equilibrium in linear systems. It can be shown [11] that if the restraint height $a$ is greater than a certain limiting value $a_{\lim }$ then the overall threshold stiffness of the system is associated with the $n_{b}$ th mode; if restraints are provided below this level, it may be assumed that the beam cannot ever develop the fully-braced condition and hence the concept of a threshold stiffness does not apply. The value of $a_{\text {lim }}$ is given by:

$$
a_{\lim }=\frac{h_{s} \kappa_{s}}{4 \sqrt{1+\kappa_{s}}},
$$

where $\kappa=L^{2} G I_{t} / \pi^{2} E I_{w}$ and $\kappa_{s}=\kappa /\left(n_{b}+1\right)^{2}$.

\section{Threshold stiffness}

Provided the restraints are positioned at a level above $a_{\lim }$ then the beam has the potential to be braced fully, the requirement for which being $K>K_{T}$. The following formula, derived on the basis of the analytical results, provides an estimate for the threshold stiffness:

$$
K_{T}=\left(\frac{E I_{z}}{s^{3}}\right) \frac{62\left(1+\kappa_{s}\right)}{A_{0}+A_{1} \hat{a}}
$$

where:

$$
\begin{gathered}
A_{0}=0.45+2.8 \nu_{b, T} \kappa_{s}, \\
A_{1}=6.3 \nu_{b, T}+2.2 \kappa_{s}-1,
\end{gathered}
$$

and the factor $\nu_{b, T}=\left\{1+\cos \left[\pi /\left(n_{b}+1\right)\right]\right\}^{-1}$. Values of $\nu_{b, T}$ for corresponding numbers of restraints are given in Table 1. Figures 3 to 5 show the close agreement between the analytical and design formula.

An axially-loaded column with a single restraint located at mid-height buckles into the second mode if the stiffness of the restraint $K>16 \pi^{2} E I_{z} / L^{3}$; this value represents the equivalent threshold stiffness. This varies from being greatly conservative to greatly unsafe across the standard ranges of $\hat{a}$ and $\kappa$ : for $\hat{a}=1$ the column rule tends to provide estimates that are twice too large; for $\hat{a}=0$, the predicted values range from being $15 \%$ too low to nearly 5.5 times too low depending on the value of $\kappa$. Percentage errors are given in Table 2.

\section{Critical moment}

If $K \geqslant K_{T}$ then the elastic critical moment of the beam is the threshold moment, $M_{T}$ :

$$
M_{T}=\frac{\pi^{2} E I_{z}}{s^{2}}\left(\frac{h_{s}}{2}\right) \sqrt{1+\kappa_{s}} .
$$


However, if it is the case that $K<K_{T}$, then the value of $M_{\mathrm{cr}}$ is reduced from this threshold amount. A conservative estimate for $M_{\mathrm{cr}}$ in such cases is provided by the linear approximation:

$$
M_{\mathrm{cr}}=M_{\mathrm{ob}}+\frac{K}{K_{T}}\left(M_{T}-M_{\mathrm{ob}}\right) .
$$

The above approximation is most accurate for a single restraint at midspan, positioned at the compression flange, as shown in Figure 6. Accuracy decreases for lower restraint heights and for greater numbers of restraints. Since there are many freely-available computer packages capable of determining the critical moment of a beam with arbitrary intermediate restraint conditions, further design guidance on the estimation of critical moments is deemed unnecessary. Moreover, it is more often the case that the restraining members are to be designed to brace the beam fully, and so the guidance provided in $\S 3$ is of greater use.

The SCI publication P093 [12], which has been updated by SCI publication P360 [13], stated that a conservative approach to the problem of beam bracing behaviour can be provided by treating the beam as an analogous column whereby the axial load $P$ is equivalent to the maximum compressive force in the flanges, $=M / h_{s}$. For an axially-loaded column braced at midspan by an elastic restraint of stiffness $K$, the critical load is given by:

$$
P_{\mathrm{cr}}=P_{E}+\frac{3}{16} K L
$$

up to a limiting stiffness of $16 \pi^{2} E I_{z} / L^{3}$, when the beam is fully braced. When compared with the analytical results of the current work, for top flange bracing, the method returns conservative values for all but the smallest beams. However, for lower restraint heights, there exist ranges of stiffnesses where the method returns conservative estimates for the critical moment and ranges where the estimates are unsafe. Hence, it can be said that application of analogous column rules is not always appropriate in the determination of critical moments.

\section{$5 \quad$ Restraint forces}

The design formulae for the restraint force ratio $F / P$, where $F$ is the force induced in the restraint and $P=M / h_{s}$ is the maximum compressive force in the beam, is obtained by exploiting the form of the analytical result of $F / P$. The function is rational in $K$, such that the graph of $F / P$ against $K$ possesses a single horizontal asymptote, $(F / P)_{\infty}$, termed the "plateau" force, and a single vertical asymptote, $K_{\infty}$, termed the "spike" stiffness:

$$
\frac{F}{P}=(F / P)_{\infty}\left(\frac{K}{K-K_{\infty}}\right) .
$$

Since the imperfection is assumed to be a half-sine wave, the restraint force also varies sinusoidally along the span of the beam; for convenience, the maximum possible force induced is determined, i.e. the force at midspan. It is initially assumed that the beam is to be designed to support load levels close to $M=M_{T}$, and the design rules outlined in the current section are based upon this conservative assumption. Methods to account for lower loading levels are treated subsequently.

\subsection{Calculation of spike stiffness}

Equation (7) is used to calculate $K_{\infty}$, except the factor $\nu_{b, T}$ is replaced with:

$$
\nu_{b, \infty}=\left\{1-\cos \left[\pi /\left(n_{b}+1\right)\right]\right\}^{-1} .
$$

It is noted that for $n_{b}=1, K_{\infty}=K_{T}$. Values of $\nu_{b, \infty}$ for calculating $K_{\infty}$ are provided in Table 3. As is the case for calculating $K_{T}$, the values of $K_{\infty}$ obtained are more conservative as $\kappa$ is increased and also for higher restraint heights, as shown by Figures 7 and 8 . Percentage errors are given in Table 4. 


\subsection{Calculation of plateau force}

Assuming again that $M=M_{T}$, the design formula is:

$$
\left(\frac{F}{P}\right)_{\infty}=5\left(\frac{e_{1}}{L}\right)\left[\frac{\sigma\left(n_{b}+1\right)}{2} \hat{a}+\frac{(1+\kappa)^{\sigma}}{5.5+4.5 n_{b}}\right]^{-1} .
$$

For $\kappa<5\left(n_{b}-1\right), \sigma=1$; otherwise the following rules can be used to calculate $\sigma$ for $\kappa \leqslant 50$ (a value that greatly exceeds the range of practical UB values):

- For $n_{b}=1, \sigma=0.9-0.01 \kappa($ but $>0.7)$.

- For $n_{b}=2, \sigma=0.99-0.006 \kappa$ (but $>0.8$ ).

- For $n_{b}=3, \sigma=1-0.004 \kappa$ (but $>0.85$ ).

- For $n_{b} \geqslant 4$ a conservative value of $\sigma=0.95$ can be taken.

It was advised in [6] that a force of $1 \%$ of the axial load in a flange may generally be assumed as the bracing-strength requirement. For restraints positioned close to the compression flange of a sufficiently high stiffness this is indeed true, but for a combination of low restraint heights and low values of $\kappa$, this is not the case, since $F / P$ can also exceed the $2.5 \%$ limit set out by BS 5950 , as clearly seen in Figures 9 to 11 . As the number of restraints provided gets larger, the effect on $F / P$ is different depending on the restraint height; the ratio reduces for larger restraint heights, but increases for restraints positioned closer to the shear centre. Percentage errors are given in Table 5.

\subsection{Modifications for $M<M_{T}$}

In practice, it is desirable to configure the bracing system so that the effects of lateral-torsional buckling can be ignored. This condition is termed "fully-restrained" design and occurs when $\bar{\lambda}_{\mathrm{LT}} \leqslant \bar{\lambda}_{\mathrm{LT}, 0}$, where $\bar{\lambda}_{\mathrm{LT}}=\sqrt{M_{\mathrm{c}, \mathrm{Rd}} / M_{\mathrm{cr}}}$ and $M_{\mathrm{c}, \mathrm{Rd}}$ is the in-plane bending resistance of the beam. Both the Eurocode and BS 5950 define $\bar{\lambda}_{\mathrm{LT}, 0}=0.4$. If it is assumed that the beam is designed to carry a load close to its in-plane bending resistance, i.e. $M \approx M_{\mathrm{c}, \mathrm{Rd}}$, and that the restraints are stiff enough so that it is fully braced, i.e. $M_{\mathrm{cr}}=M_{T}$, then $M / M_{T}=\bar{\lambda}_{\mathrm{LT}, 0}^{2}=0.16$. Thus, in the sake of economy, the design formulae should take values of $M<M_{T}$ into account.

\section{Modification of spike stiffness}

A quick but conservative modification can be made by simply scaling $K_{\infty, M=M_{T}}$ by $M / M_{T}$. This increases in accuracy with increasing $n_{b}$, especially for low values of $\kappa$ and $n_{b} \geqslant 4$.

However, if greater accuracy is desired then the following modification factor can be applied to $K_{\infty}$ with only moderately conservative results:

$$
\frac{K_{\infty}}{K_{\infty, M=M_{T}}}=\frac{M-M_{\mathrm{ob}}}{M_{T}-M_{\mathrm{ob}}}
$$

If $M<M_{\mathrm{ob}}$, then $K_{\infty}<0$ and for $K>0,0<F / P<(F / P)_{\infty}$, owing to the function $F / P$ passing through the origin. Hence, where $M<M_{\mathrm{ob}}$, the value of $F / P$ corresponding to a stiffness $K \geqslant K_{T}$ can be taken as being equal to $(F / P)_{\infty}$, and the actual value of $K_{\infty}$ need not be calculated.

\section{Modification of plateau force}

For $\hat{a}=0$, an approximation for $(F / P)_{\infty}$ can be found by simply scaling the value found in $\S 5.2$ by $M / M_{T}$. For $\hat{a}=1$, simply using the value of $(F / P)_{\infty, M=M_{T}}$ provides only slightly conservative results, with accuracy improving for higher restraint values and low values of $\kappa$. If $\hat{a} \leqslant 0.15 \kappa / n_{b}$, greater economy can be achieved by using a scaling factor of $\sqrt{M / M_{T}}$ i.e. $(F / P)_{\infty}=\sqrt{M / M_{T}}(F / P)_{\infty, M=M_{T}}$. 


\subsection{Comparison with equivalent column rule}

In $§ 5.2$ it was shown how the blanket rule of a $1 \%$ restraint force ratio suggested in [6] for beams was adequate, if somewhat conservative for restraints positioned closer to the compression flange. For lower restraint heights, this rule and also the BS 5950 rule of $2.5 \%$, which are based on column buckling theory, have been shown to underestimate the actual restraint force ratio by a considerable margin. Expressions are provided in [7] and [14] for the restraint force ratio for a simply-supported axially-loaded strut for the cases of a single restraint attached at an arbitrary point along the span of the strut, restraints attached at third points and multiple restraints. For the case of a single restraint at midspan the following expression is given:

$$
\frac{F}{P}=\frac{e_{1}}{L} \frac{1}{\frac{P_{E}}{P}-1}\left[\frac{P_{E}}{K L} \frac{P}{P_{E}}+\frac{1}{2 \pi} \sqrt{\frac{P_{E}}{P}} \tan \left(\frac{\pi}{2} \sqrt{\frac{P}{P_{E}}}\right)-\frac{1}{4}\right]^{-1} .
$$

For two restraints, the force $F$ in each restraint is given by:

$$
\frac{F}{P}=\frac{\sqrt{3}}{2} \frac{e_{1}}{L} \frac{1}{\frac{P_{E}}{P}-1}\left[\frac{P_{E}}{K L} \frac{P}{P_{E}}+\frac{\sin \left(\frac{\pi}{3} \sqrt{\frac{P}{P_{E}}}\right)}{\pi \sqrt{\frac{P}{P_{E}}}\left[2 \cos \left(\frac{\pi}{3} \sqrt{\frac{P}{P_{E}}}\right)-1\right]}-\frac{1}{3}\right]^{-1} .
$$

Corresponding equations for $(F / P)_{\infty}$ are obtained by setting $P_{E} / K L=0$ in the denominator. Given a particular nondimensional restraint height $\hat{a}$, it is possible to find at what value of $\kappa$ the value of $(F / P)_{\infty}$, as calculated by the column rules, is equal to the corresponding value calculated using the beam rules of the current work. This value is denoted $\kappa_{\lim }$. If $\kappa<\kappa_{\lim }$ then the column rules provide unsafe values; if $\kappa>\kappa_{\lim }$ then the column rules provide conservative values. For the two cases above, values for $\hat{a}=0$ and $\hat{a}=1$ are given in Table 6 , along with an almost exact formula for restraint heights in between. As can be seen, the rules provide unsafe values for typical UB sections $(1 \leqslant \kappa \leqslant 15)$ when restrained at the shear centre, and overly conservative values when restraint is provided at the compression flange.

For $n_{b}>2$, following the approach of the Eurocode, the total sum of the restraint forces, $\sum F_{i}$, can be represented by an equivalent UDL, $q_{F}$, such that $\sum F_{i}=q_{F} L$, with the restraint forces "smeared" as an equivalent continuous restraint of stiffness $k$. The following formula for the magnitude of $q_{F}$ was provided:

$$
\frac{q_{F} L}{P}=\frac{8}{\pi^{2}} \frac{k L^{2}}{P_{E}} \frac{e_{1}}{L}\left[1+\frac{k L^{2}}{\pi^{2} P_{E}}-\frac{P}{P_{E}}\right]^{-1} .
$$

The approach of the Eurocode of representing the initial imperfection in the main members by an equivalent horizontal force in the restraining system was then applied. In the context of the current investigation, this is equivalent to $q_{F}$ and it is related to the initial imperfection of the main member and the maximum lateral deflection of the restraining system, $\delta_{q}$ :

$$
\frac{q_{F} L}{P}=\frac{8\left(e_{1}+\delta_{q}\right)}{L} .
$$

The value of $\delta_{q}=f_{\max } / k$, where $f$ is the distributed restraint force. Since the initial imperfection is assumed to be in the form of a half-sine wave, the restraint forces also vary sinusoidally, i.e. $f=f_{\max } \sin (\pi x / L)$. Since the sinusoidally varying restraint force is now being represented by an equivalent UDL, for the moments at midspan to be equivalent, $q_{F}=8 f_{\max } / \pi^{2}$. Examining the derivation of restraint forces in the current work again, as $f_{\max }$ and $F_{\max }$ are both calculated based on the restraint compression function $X=F / K=f / k$ at a particular point and that $p$ is merely $P$ scaled by $1 / L, f_{\max } / p$ can be rescaled to $F / P$, since it is assumed that the maximum discrete restraint force is being designed for. It is also noted that for $n_{b}$ discrete restraints of stiffness $K$, the equivalent continuous stiffness $k=n_{b} K / L$. Hence the Eurocode formula can be recast as:

$$
\frac{F}{P}=\pi^{2} \frac{e_{1}}{L}\left[1-\frac{\pi^{2}}{n_{b}} \frac{P}{P_{E}} \frac{P_{E}}{K L}\right]^{-1}
$$


If the limit of Equation (21) as $K \rightarrow \infty$ is taken, then a value of $(F / P)_{\infty}=1.97 \%$ is obtained for $e_{1} / L=1 / 500$. A comparison with Figure 11 shows that for higher restraint heights, this rule is overly conservative, while for a combination of low restraint heights and low values of $\kappa$ the Eurocode rule is unsafe. Moreover, as the number of restraints is increased, this effect is exacerbated.

\section{Optimisation of stiffness and strength requirements}

In the case where bracing members are attached to a system of primary beams orthogonally, then the stiffness of the bracing members is given by $K=E A_{b} / L_{b}$, where the subscript $b$ denotes properties of the bracing member. If it is assumed that the bracing members only act in tension then the strength, or resistance, of the brace is given by $F=A_{b} f_{\mathrm{y}}$. This implies that only bracing members on a particular side act to restrain the beam when it deflects. The design of the bracing members can be optimised by equating the area demands for both properties, i.e. strength and stiffness, resulting in the following expression for the cross-sectional areas of the bracing members:

$$
A_{b}=\frac{K_{\infty} L_{b}}{E}+\frac{M}{h_{s} f_{\mathrm{y}}}\left(\frac{F}{P}\right)_{\infty} .
$$

It should be noted, however, that this formula can return values for $A_{b}$ that correspond to stiffnesses that are less than $K_{T}$; since the formula is based on finding the ratio of $K / K_{\infty}$ at which the area demands are equivalent. This condition occurs when:

$$
K_{T}>K_{\infty}+\left(\frac{F}{P}\right)_{\infty} \frac{P E}{L_{b} f_{\mathrm{y}}} .
$$

There are numerous factors that influence the minimum value of $K_{T}$ for when this condition occurs, and thus finding a convenient rule is inhibited. It suffices to say that, in general, the condition prevails for (i) a high number of restraints, (ii) high values of $L / L_{b}$ i.e. for relatively short brace lengths, and (iii) low values of $M / M_{T}$. Generally, the condition does not prevail for high values of $\hat{a}$. In cases where the condition is satisfied, restraints of stiffness $K \geqslant K_{T}$ are able to resist the load induced in them, and thus the design value of $A_{b}$ should be based on satisfying the stiffness demand. Alternatively, in the interest of convenience, a maximum level of restraint force can be specified and the corresponding value of $K$ can be found using the relation presented in $\S 5$.

\section{Conclusions}

Formulae for the design of beams with lateral restraints and the design of the bracing members themselves have been provided, based on analytical results derived from a linear Rayleigh-Ritz analysis of the system. It has been demonstrated that the design rules agree closely with the analytical results. These have also been compared with design rules developed based on analogies with column behaviour, whereupon it was found that the column rules provided results that were overly conservative for restraints positioned close to the compression flange of the beam, and unsafe for restraints positioned close to the shear centre of the beam. A procedure has been developed to optimise the design of bracing members by taking both stiffness and strength into account.

\section{Acknowledgements}

The work was partially funded by the UK Engineering and Physical Sciences Research Council through project grant: EP/F022182/1, and also by the Department of Civil and Environmental Engineering at Imperial College London. 


\section{References}

[1] S. P. Timoshenko and J. M. Gere, Theory of elastic stability. New York, USA: McGraw-Hill, 2nd ed., 1961.

[2] British Standards Institute, BS 5950-1:2000 Structural use of steelwork in building - Part 1: Code of practice for design - Rolled and welded sections. London, UK: British Standards Institute, 2000.

[3] American Institute of Steel Construction, Steel Constructors Manual. Chicago, USA: American Institute of Steel Construction, 13th ed., 2005.

[4] J. A. Yura and B. Phillips, "Bracing requirements for elastic steel beams," Tech. Rep. No. 1239-1, Center for Transportation Research, University of Texas at Austin, May 1992.

[5] Comité Européan de Normalisation, EN1993-1-1: Eurocode 3: Design of steel structures Part 1-1: General rules and rules for buildings. London, UK: British Standards Institute, 2005.

[6] Y. C. Wang and D. A. Nethercot, "Bracing requirements for laterally unrestrained beams," Journal of Constructional Steel Research, vol. 17, pp. 305-15, 1990.

[7] F. A. N. Al-Shawi, "Determination of restraint forces for steel struts," Proceedings of the Institution of Civil Engineers - Structures and Buildings, vol. 128, pp. 282-9, August 1998.

[8] J. A. Yura, "Fundamentals of beam bracing," Engineering Journal, American Institute of Steel Construction, vol. 38, pp. 11-26, First quarter 2001.

[9] N. Trahair, Flexural-torsional buckling of structures. London, UK: E \& FN SPON, 1993.

[10] V. Z. Vlasov, Thin-walled elastic beams. Jerusalem, Israel: Israel Program for Scientific Translations, 2nd ed., 1961.

[11] F. McCann, Stability of beams with discrete lateral restraints. PhD thesis, Imperial College London, 2012.

[12] D. A. Nethercot and R. M. Lawson, Lateral stability of steel beams and columns - Common cases of restraint. Ascot, UK: Steel Construction Institute, 1992. SCI publication P093.

[13] L. Gardner, Stability of steel beams and columns: in accordance with Eurocodes and the UK National Annexes. Ascot, UK: Steel Construction Institute, 2011. SCI publication P360.

[14] F. A. N. Al-Shawi, "Stiffness of restraint for steel struts with elastic end supports," Proceedings of the Institution of Civil Engineers - Structures and Buildings, vol. 146, no. 2, pp. 153-9, 2001. 
Captions for figures

Figure 1: Cross-sectional geometry, system axes and configuration of the analytical model.

Figure 2: Typical critical mode progression for beams with discrete restraints.

Figure 3: A comparison of actual and approximate values of $K_{T}$ for $n_{b}=1$.

Figure 4: A comparison of actual and approximate values of $K_{T}$ for $n_{b}=2$.

Figure 5: A comparison of actual and approximate values of $K_{T}$ for $n_{b}=3$.

Figure 6: A comparison of actual and approximated values of $M_{\mathrm{cr}}$ for $n_{b}=1$ and $\hat{a}=1$.

Figure 7: A comparison of actual and approximated values of $K_{\infty}$ for $n_{b}=2$ and $M=M_{T}$.

Figure 8: A comparison of actual and approximated values of $K_{\infty}$ for $n_{b}=3$ and $M=M_{T}$.

Figure 9: A comparison of actual and approximated values of $(F / P)_{\infty}$ for $n_{b}=1$ and $M=M_{T}$.

Figure 10: A comparison of actual and approximated values of $(F / P)_{\infty}$ for $n_{b}=2$ and $M=M_{T}$.

Figure 11: A comparison of actual and approximated values of $(F / P)_{\infty}$ for $n_{b}=3$ and $M=M_{T}$. 
Table 1: Values of $\nu_{b, T}$ used for calculating $K_{T}$.

Table 2: Maximum, minimum and average percentage errors between actual and approximate values of $\gamma_{s, T}$ as shown in Figures 3 to 5 . Negative errors correspond to ranges of restraint height where the approximate formula underestimates the threshold stiffness.

Table 3: Values of $\nu_{b, \infty}$ used for calculating $K_{\infty}$.

Table 4: Maximum, minimum and average percentage errors between actual and approximate values of $\gamma_{s, \infty}$ as shown in Figures 7 and 8. Negative errors correspond to ranges of restraint heights where the approximate formula underestimates the actual value.

Table 5: Maximum, minimum and average percentage errors between actual and approximate values of $(F / P)_{\infty}$ for as shown in Figures 9 to 11. Negative errors correspond to ranges of restraint heights where the approximate formula underestimates the actual value.

Table 6: Values of $\kappa_{\text {lim }}$. 


\begin{tabular}{cccc}
\hline \hline$n_{b}$ & $\nu_{b, T}$ & $n_{b}$ & $\nu_{b, T}$ \\
\hline 1 & 1.000 & 6 & 0.526 \\
2 & 0.667 & 7 & 0.520 \\
3 & 0.586 & 8 & 0.516 \\
4 & 0.553 & 9 & 0.513 \\
5 & 0.536 & 10 & 0.510 \\
\hline$n_{b} \rightarrow \infty$ & $\nu_{b, T} \rightarrow 0.500$ \\
\hline
\end{tabular}

Table 1: Table 1

\begin{tabular}{cccccccccc}
\hline \hline & \multicolumn{3}{c}{$\kappa=1$} & \multicolumn{3}{c}{$\kappa=7$} & \multicolumn{3}{c}{$\kappa=25$} \\
\hline$n_{b}$ & Max & Min & Avg & Max & Min & Avg & Max & Min & Avg \\
\hline 1 & 11.7 & 2.8 & 7.3 & 10.3 & 5.8 & 8.0 & 9.6 & -0.9 & 3.0 \\
2 & 13.3 & 1.2 & 6.8 & 6.3 & -0.4 & 2.4 & 5.1 & -6.0 & -2.8 \\
3 & 15.6 & 1.2 & 7.8 & 8.5 & -2.7 & 2.5 & 1.2 & -4.2 & -3.2 \\
\hline
\end{tabular}

Table 2: Table 2

\begin{tabular}{cccc}
\hline \hline$n_{b}$ & $\nu_{b, \infty}$ & $n_{b}$ & $\nu_{b, \infty}$ \\
\hline 2 & 2.000 & 7 & 13.14 \\
3 & 3.414 & 8 & 16.58 \\
4 & 5.236 & 9 & 20.43 \\
5 & 7.646 & 10 & 24.69 \\
6 & 10.10 & 11 & 29.35 \\
\hline
\end{tabular}

Table 3: Table 3

\begin{tabular}{cccccccccc}
\hline \hline & \multicolumn{3}{c}{$\kappa=1$} & \multicolumn{3}{c}{$\kappa=7$} & \multicolumn{3}{c}{$\kappa=25$} \\
\hline$n_{b}$ & Max & Min & Avg & Max & Min & Avg & Max & Min & Avg \\
\hline 2 & 7.6 & 4.0 & 5.6 & 17.6 & 0.9 & 11.8 & 20.5 & 2.6 & 13.6 \\
3 & 7.0 & 3.5 & 4.1 & 15.2 & 2.3 & 11.4 & 26.6 & 2.9 & 17.9 \\
\hline
\end{tabular}

Table 4: Table 4

\begin{tabular}{cccccccccc}
\hline \hline & \multicolumn{1}{c}{$\kappa=1$} & \multicolumn{3}{c}{$\kappa=7$} & \multicolumn{3}{c}{$\kappa=25$} \\
\hline$n_{b}$ & Max & Min & Avg & Max & Min & Avg & Max & Min & Avg \\
\hline 1 & 4.9 & -2.8 & 0.3 & 2.0 & -0.4 & 0.4 & -14.6 & -16.0 & -15.6 \\
2 & 5.2 & -3.8 & 2.0 & 2.1 & 0.4 & 1.0 & -3.0 & -6.7 & 5.8 \\
3 & 2.3 & -6.2 & 0.0 & 3.1 & 1.7 & 2.2 & -2.3 & -5.5 & -4.8 \\
\hline
\end{tabular}

Table 5: Table 5 


\begin{tabular}{ccc}
\hline \hline & $n_{b}=1$ & $n_{b}=2$ \\
\hline$\hat{a}=0$ & 16.9 & 52.6 \\
$0<\hat{a}<1$ & $16.9-24.7 \hat{a}+8.4 \hat{a}^{2}$ & $52.6-74.5 \hat{a}+26.0 \hat{a}^{2}$ \\
$\hat{a}=1$ & 0.6 & 4.1 \\
\hline
\end{tabular}

Table 6: Table 6 

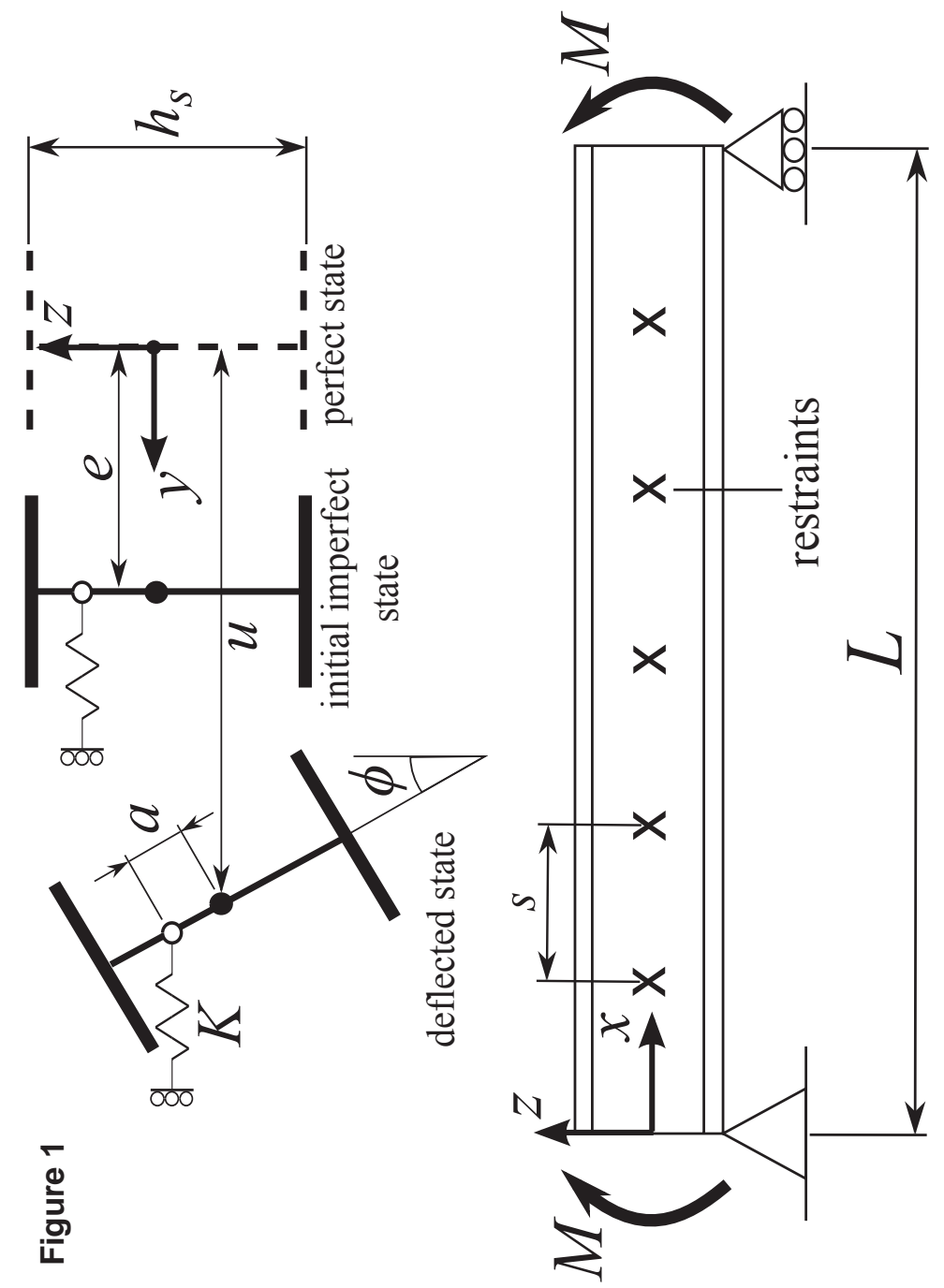


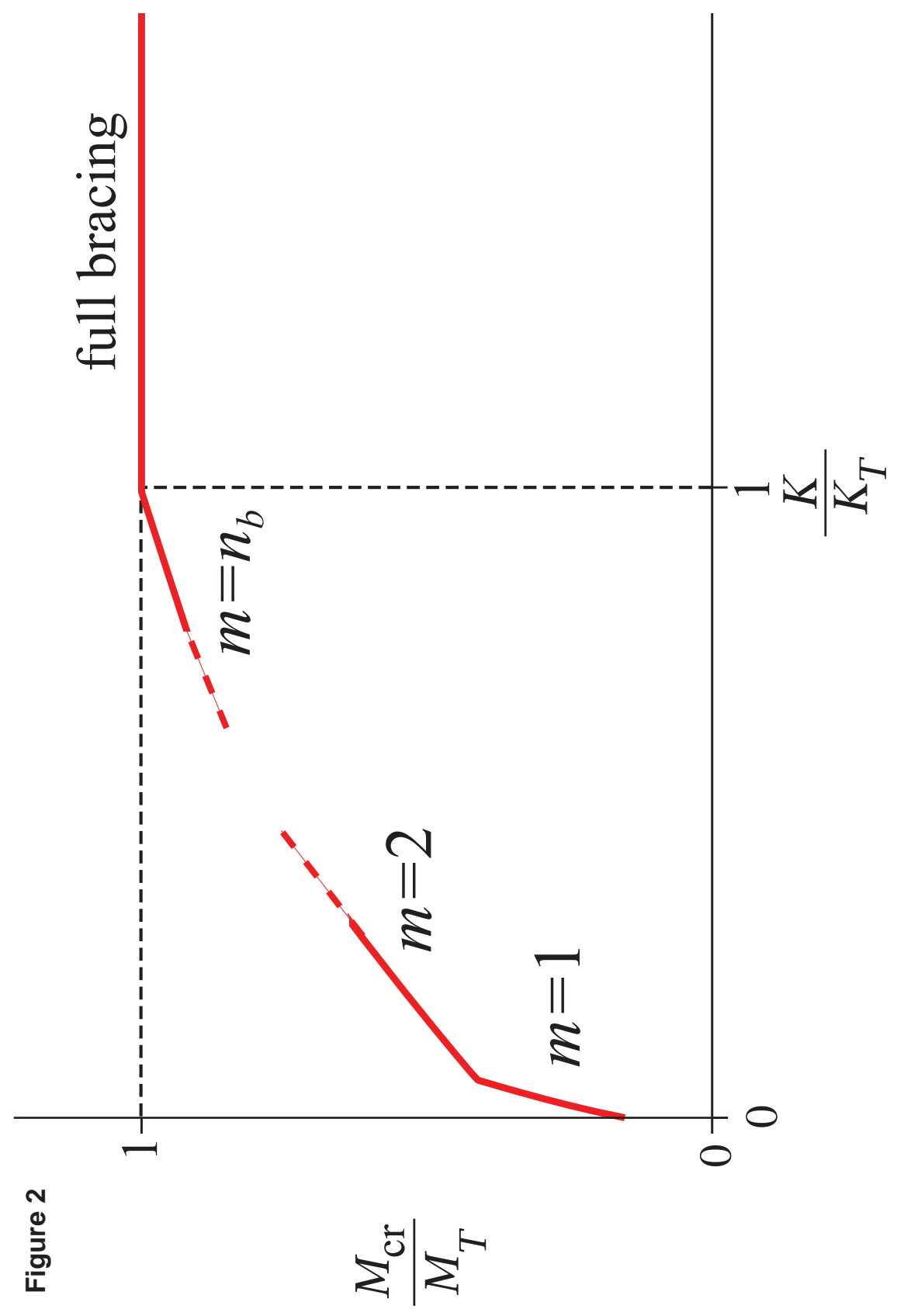




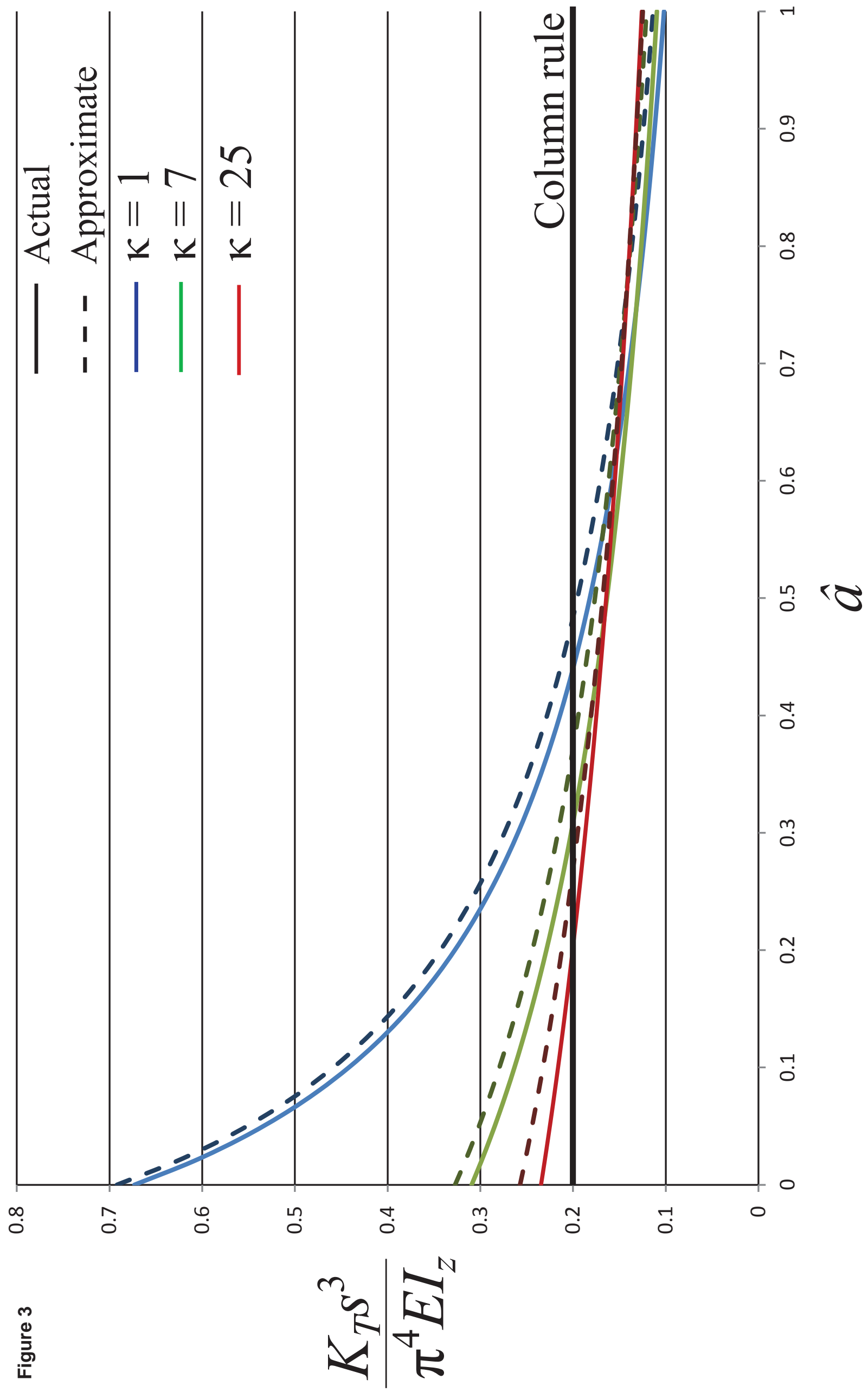




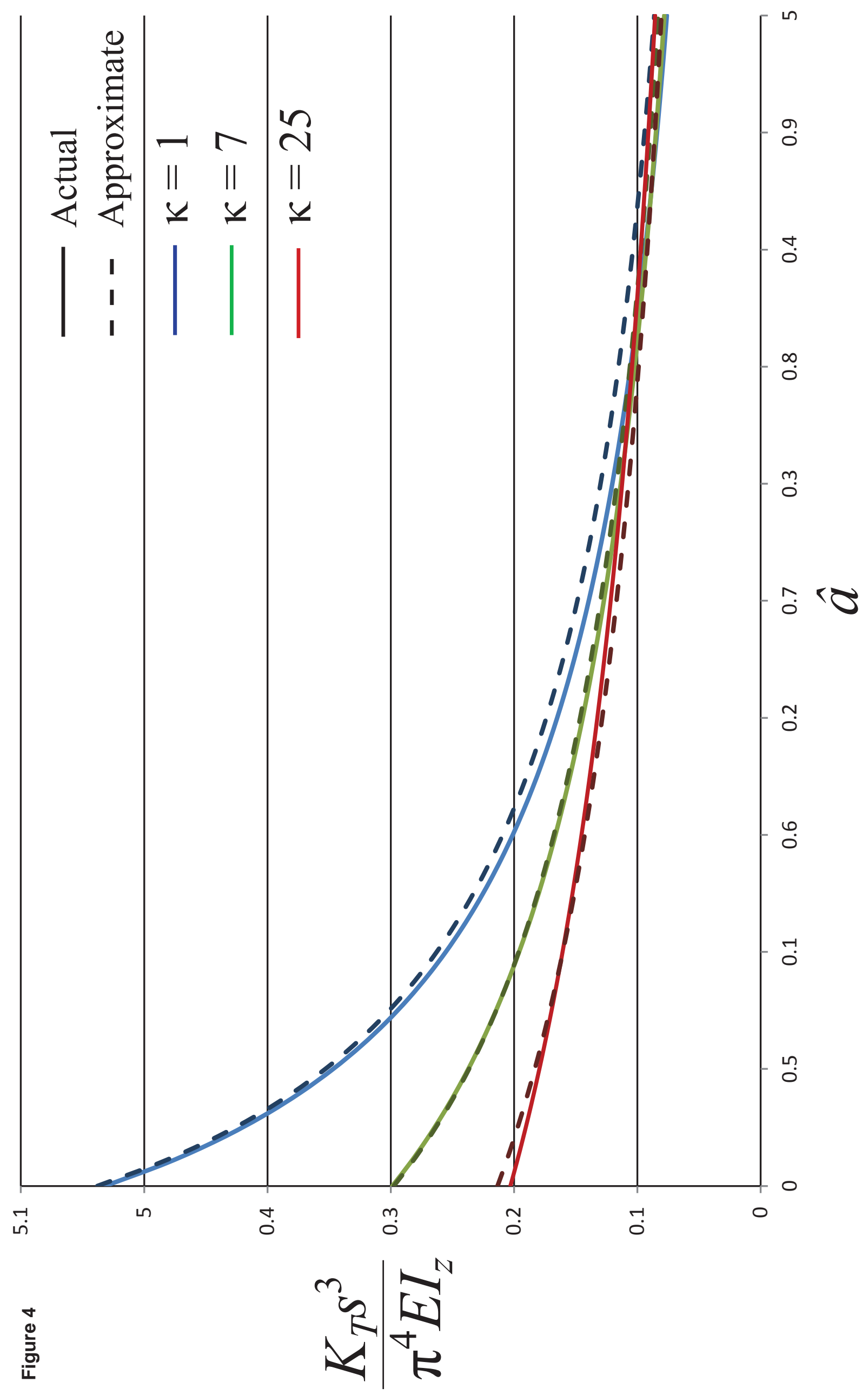




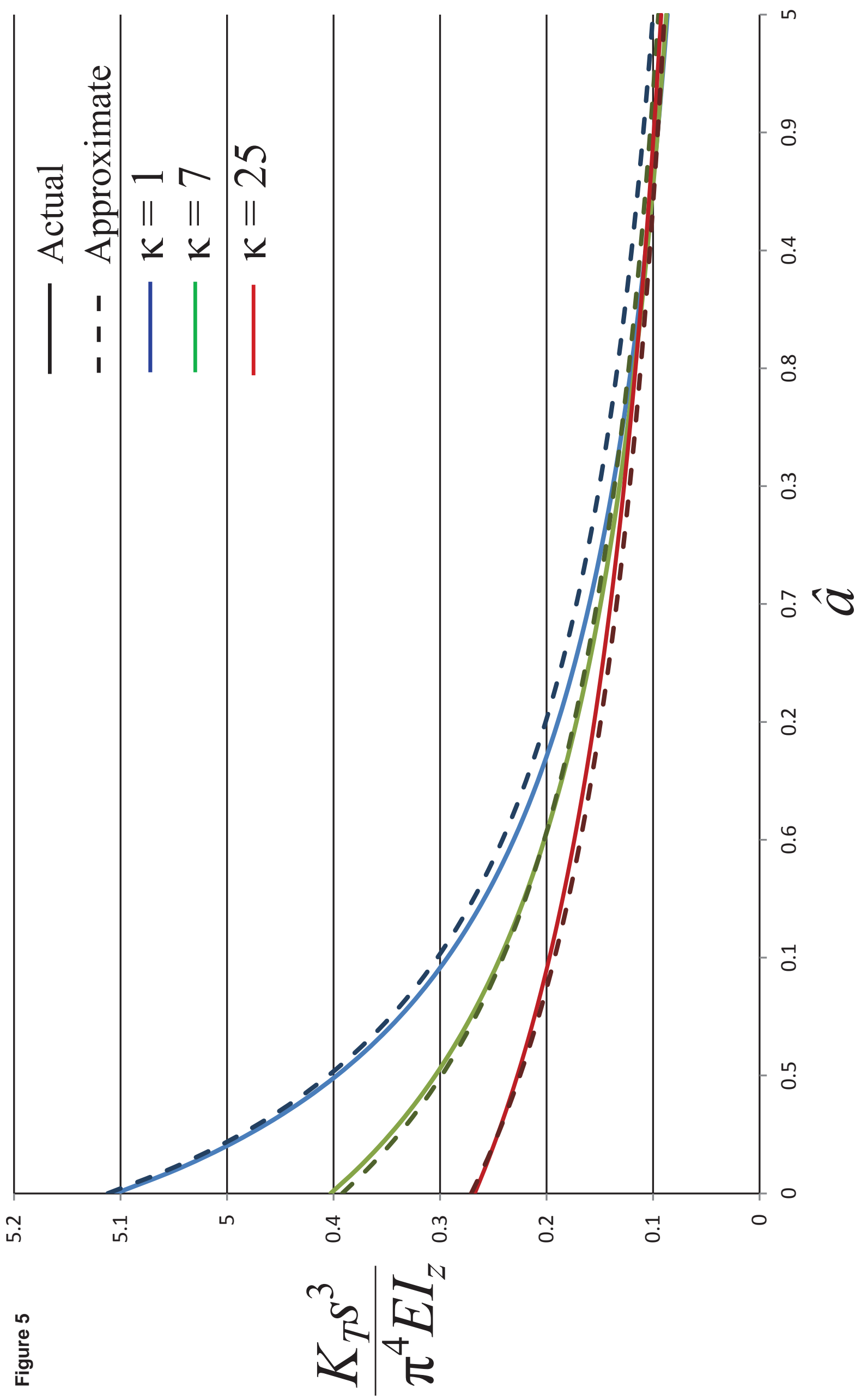




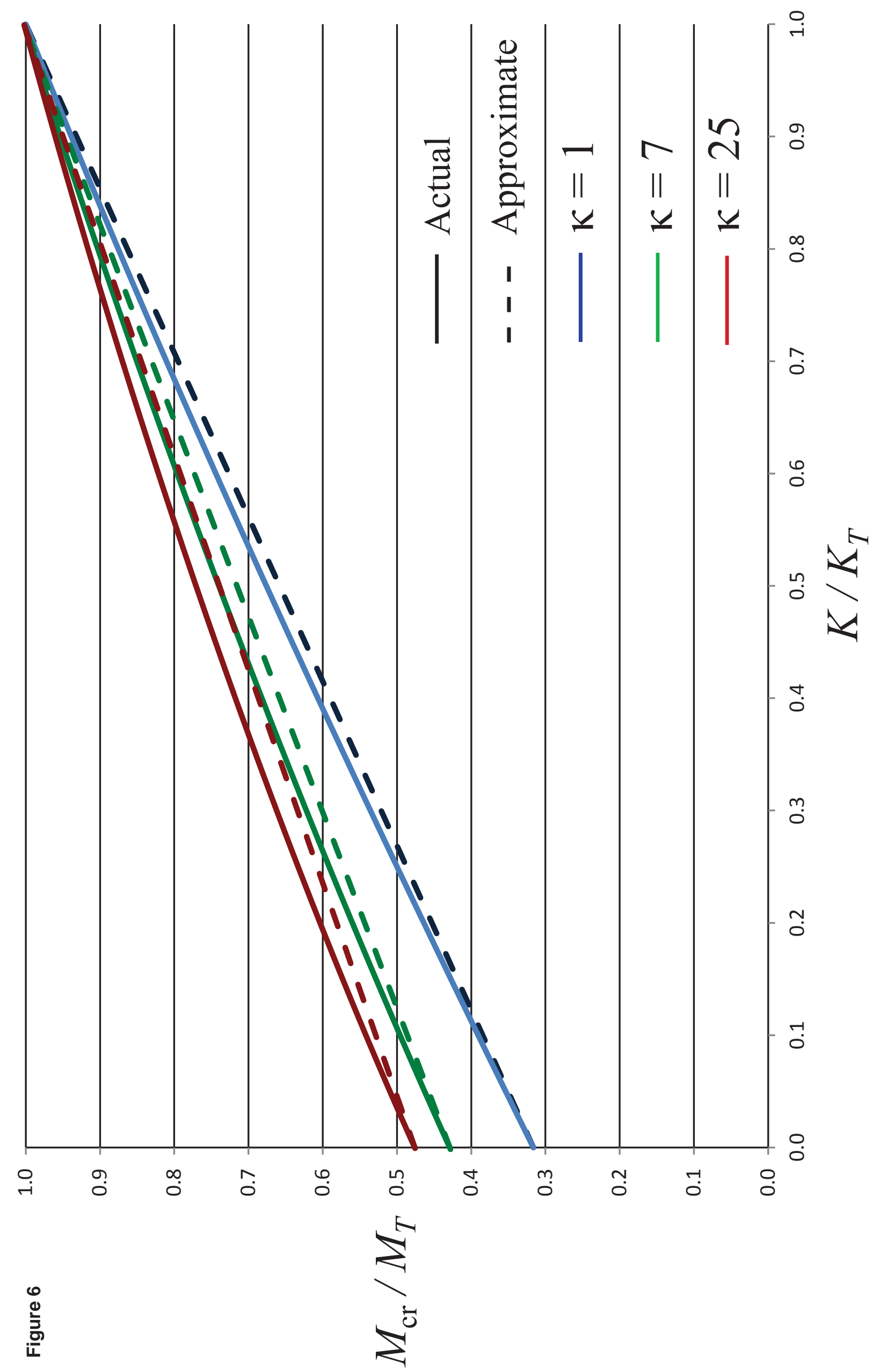




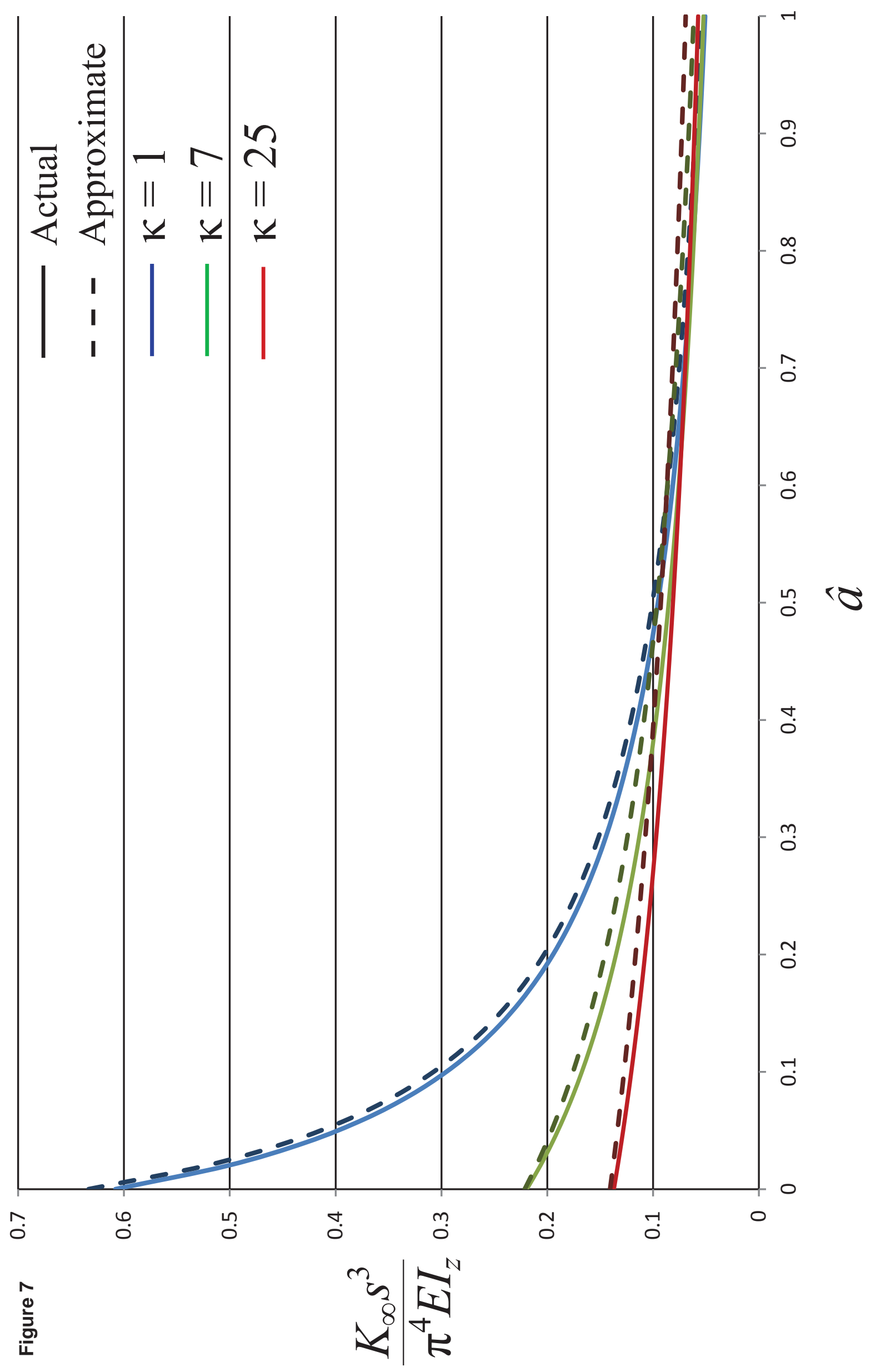




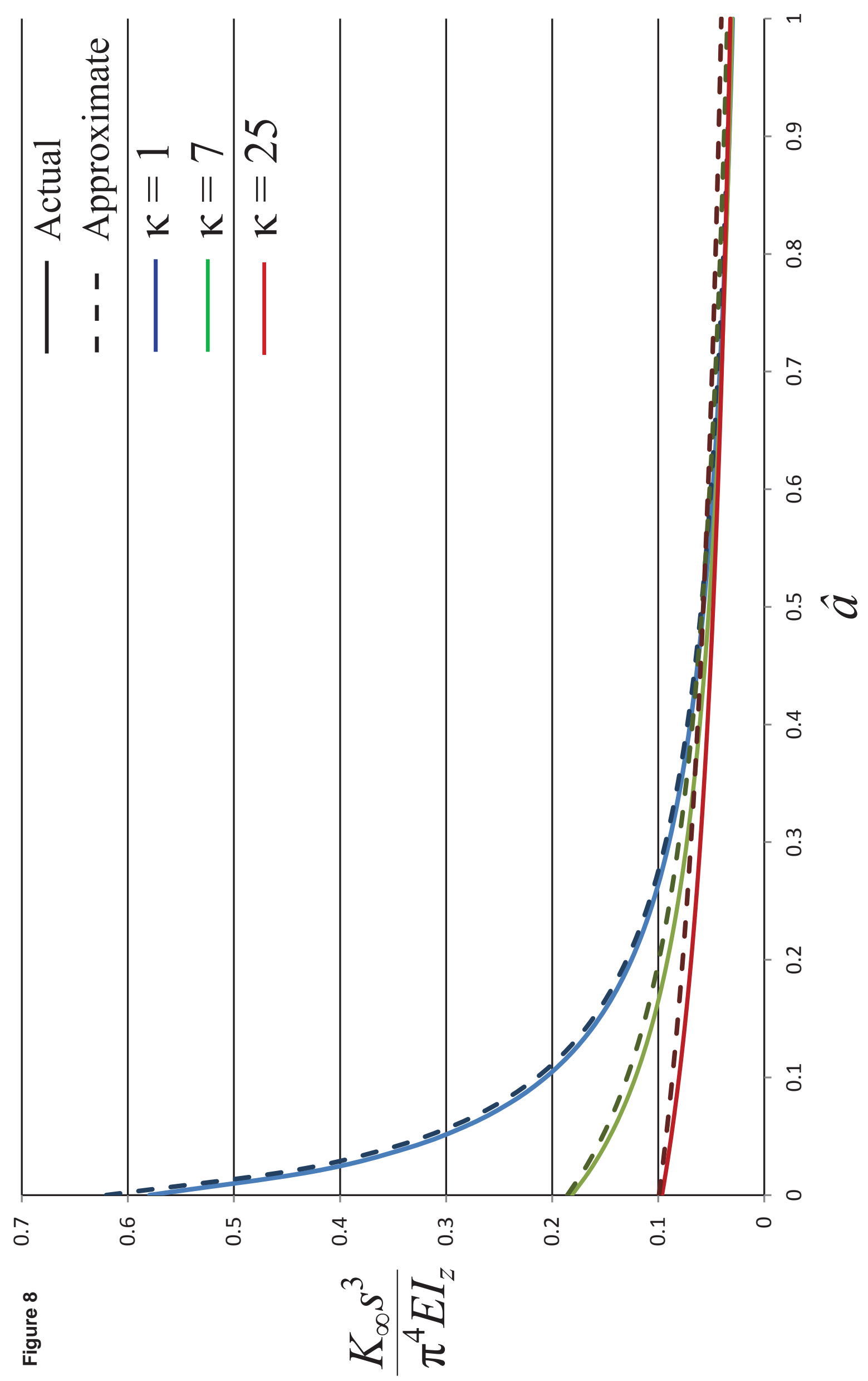




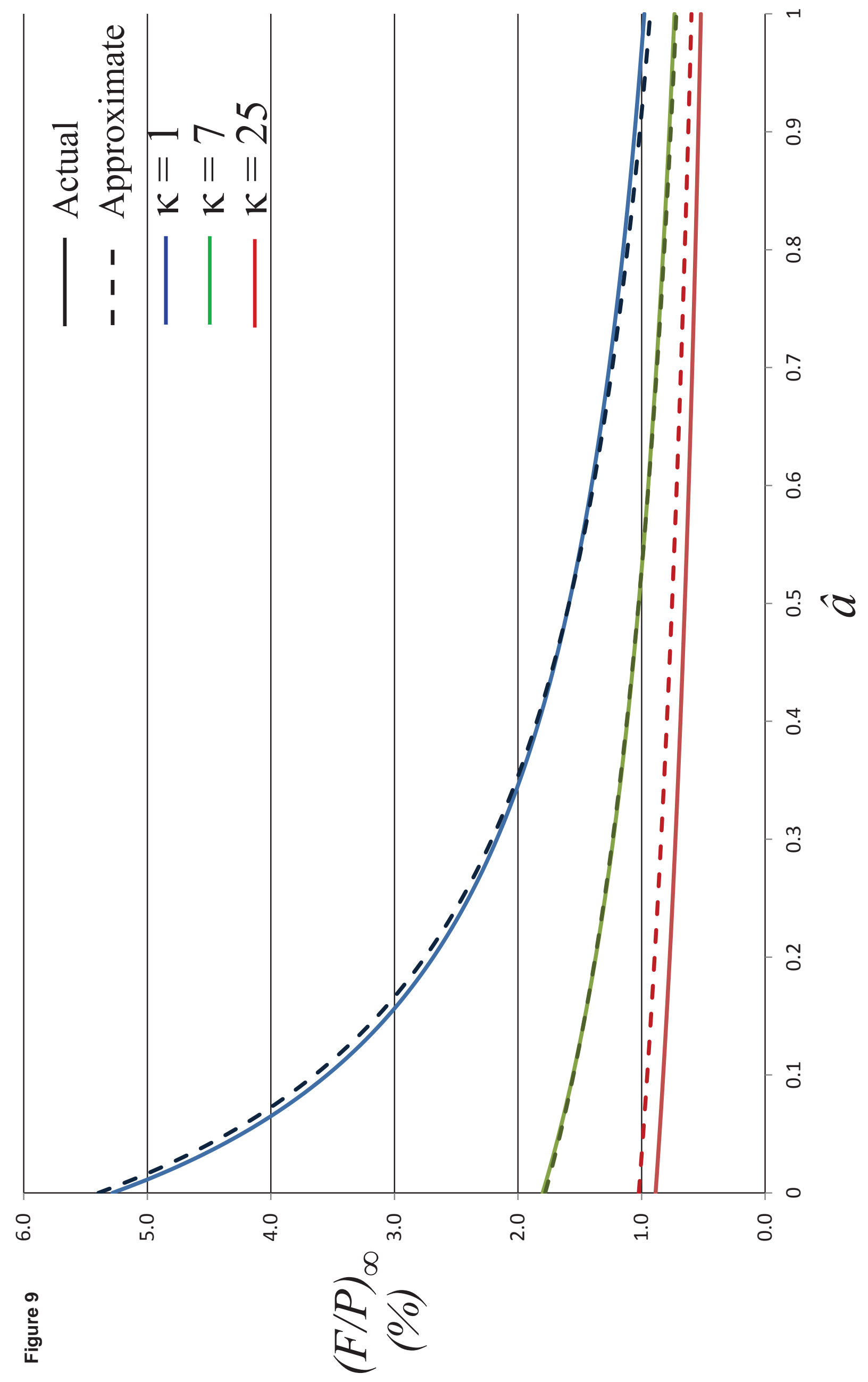




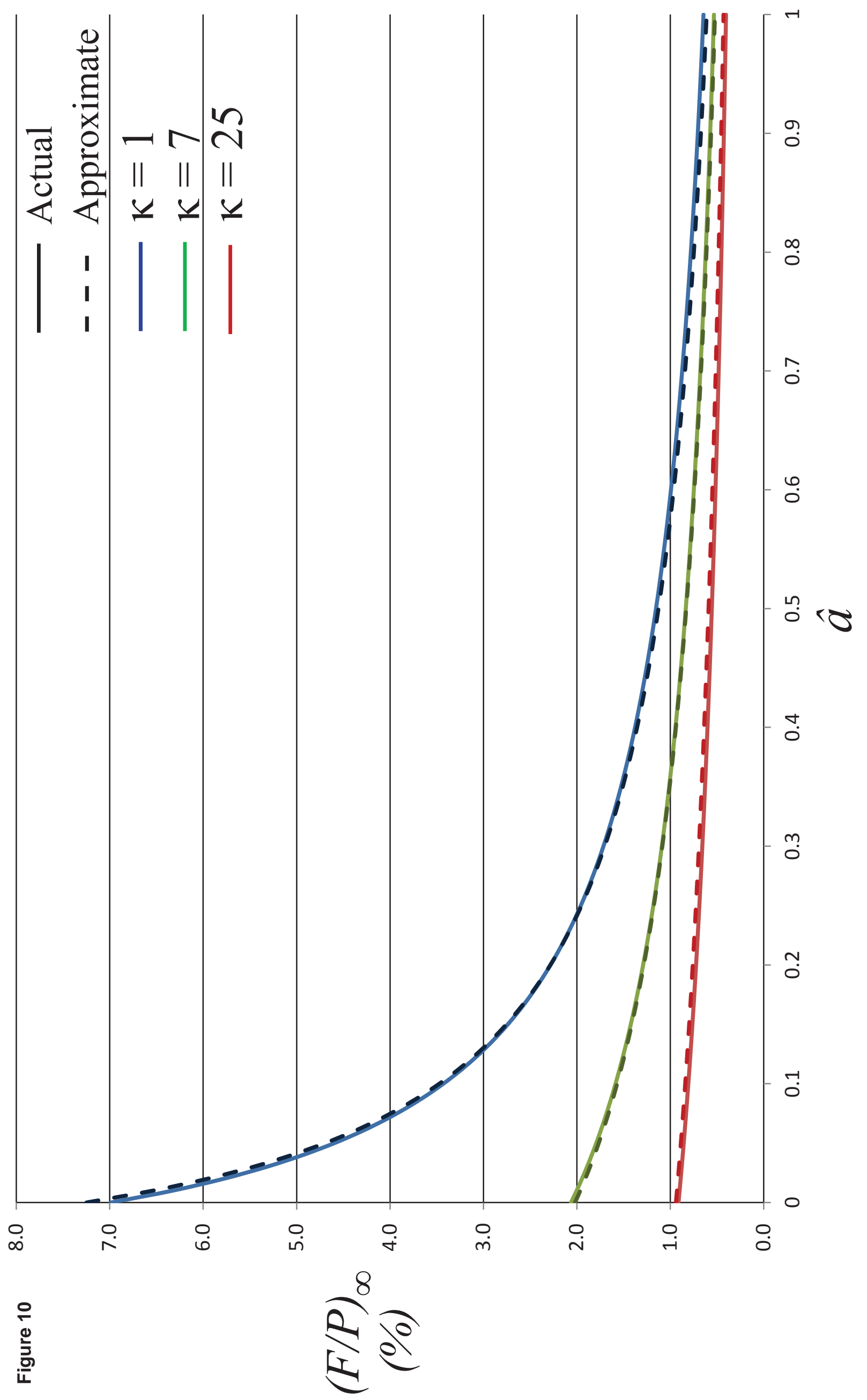




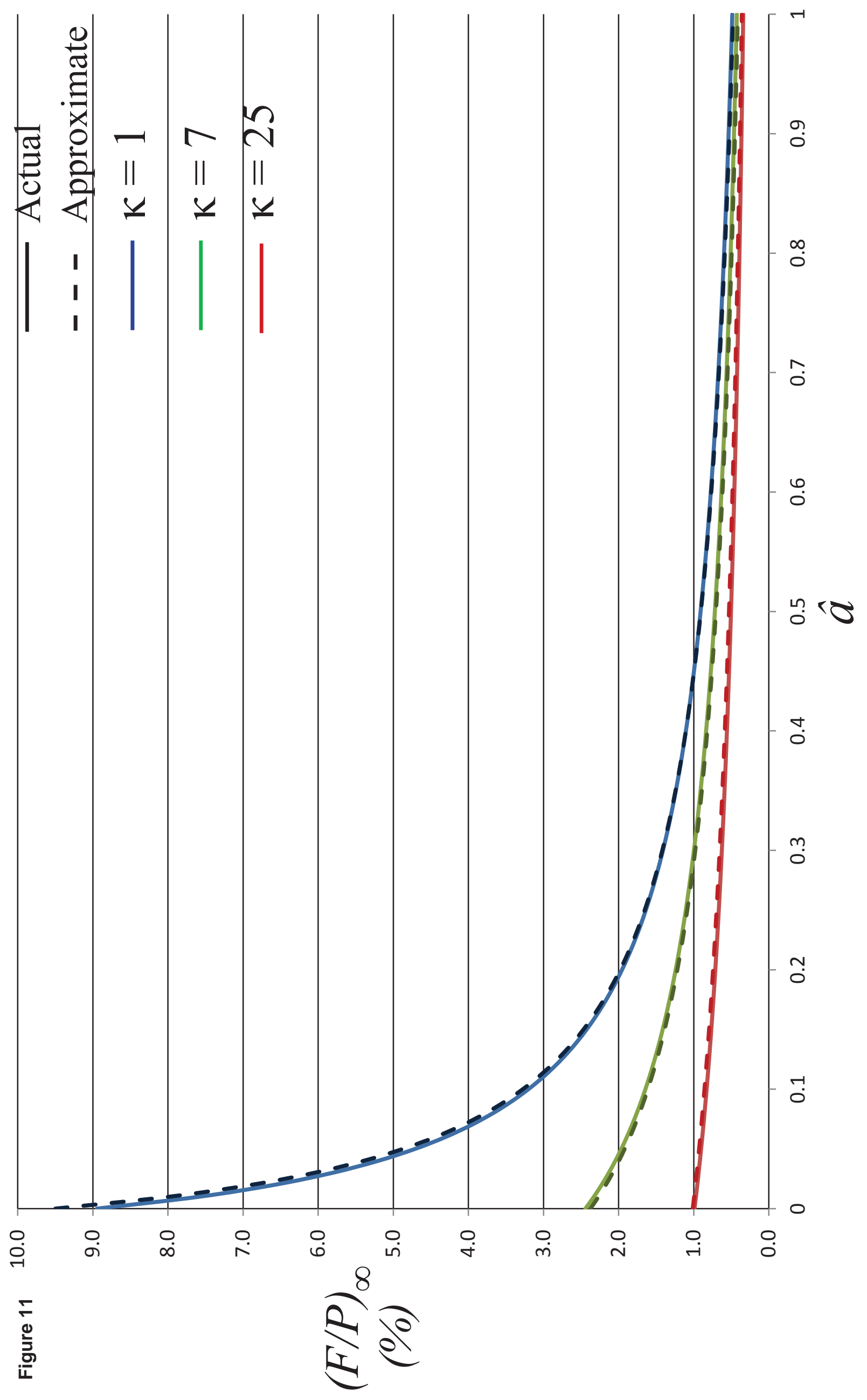

